\title{
Changes in cerebral blood flow in rheumatology patients treated with rituximab
}

\author{
Uygar Utku' ${ }^{1}$ Gözde Çetin², Nurhan Atilla ${ }^{3}$ \\ ${ }^{1}$ Department of Neurology, Kahramanmaraş Sütçü İmam University School of Medicine, Kahramanmaraş, Turkey \\ ${ }^{2}$ Department of Rheumatology, Kahramanmaraş Sütçü İmam University School of Medicine, Kahramanmaraş, Turkey \\ ${ }^{3}$ Department of Pulmonology, Kahramanmaraş Sütçü Imam University School of Medicine, Kahramanmaraş, Turkey
}

DOI: $10.18621 /$ eurj.346321

\begin{abstract}
Objectives: We aimed to assess the effects of rituximab therapy on cerebral hemodynamics in rheumatic patients with transcranial Doppler (TCD) ultrasonography.

Methods: The study included seven patients aged 20-65 years (2 patients with secondary interstitial lung involvement to rheumatoid arthritis, 3 patients with systemic lupus erythematosus and lupus nephritis, 2 patients with scleroderma and secondary interstitial lung disease) who received rituximab treatment due to rheumatic diseases. Healthy control group (non-rheumatic group) consisted of seven age and sex-matched, randomly selected persons did not have risk factors for atherosclerosis. Bilateral middle cerebral artery peak-systolic, end-diastolic, and mean blood flow velocities, Gosling's pulsatility index values, and Pourcelot's resistance index values were recorded with TCD by a neurosonologist before rituximab administration. Control TCD performed to the rheumatic group after six months from the last rituximab dosage.

Results: One patient was male and six patients were female. Peak-systolic, end-diastolic and mean blood flow velocities of rheumatic patients were significantly higher than healthy group $(p<0.001)$. Peak-systolic, enddiastolic and mean blood flow velocities of rheumatic patients were significantly decreased after rituximab therapy $(p<0.001)$.

Conclusions: This study highlights that the increased cerebral blood flow is indirectly associated with atherosclerosis regarding persistent inflammation in patients with rheumatic diseases. It was thought that treatment with rituximab could reverse this situation.
\end{abstract}

Keywords: Rituximab, inflammation, atherosclerosis, cerebral blood flow

Received: October 24, 2017; Accepted: January 22, 2018; Published Online: March 8, 2018

$I^{\prime}$ nflammation has an important role in the pathogenesis and prognosis of atherosclerosis [1, 2]. Rituximab is a monoclonal antibody drug used for improving the symptoms and preventing from the rheumatologic diseases [3]. Apart from traditional vasculo-protective agents, biologics such as rituximab, infliximab and etanercept may also exert favorable effects on the atherosclerosis and arterial stiffness. Furthermore, none of the existing studies examined with transcranial Doppler (TCD) ultrasonography whether increased arterial stiffness can be reversed by anti-inflammatory therapy. The technique of TCD ultrasonography enables assessment of hemodynamic parameters, including flow velocity in intracranial

Address for correspondence: Uygar Utku, MD., Assistant Professor, Kahramanmaraş Sütçü Imam University School of Medicine, Department of Neurology, 46100 Kahramanmaraş, Turkey

E-mail: utkuzar@gmail.com 
arteries. A milder or more generalized increased flow velocity could reflect intracerebral atherosclerosis. Here, we aimed to assess the effects of rituximab treatment on inflammation regarding atherosclerosis in rheumatic patients with TCD ultrasonography.

\section{METHODS}

Seven patients aged 20-65 years (2 patients with secondary interstitial lung involvement to rheumatoid arthritis, 3 patients with systemic lupus erythematosus and lupus nephritis, 2 patients with scleroderma and secondary interstitial lung disease) were eligible for enrolment. Patients were treated with rituximab according to medical practitioners' opinion. They received two intra-venous infusions of rituximab (1000 mg each) 2 weeks apart. They have not any risk factors for atherosclerosis, such as diabetes, hypertension, and coronary artery disease. Seven healthy persons were included to the study for control group.

Firstly, TCD performed before rituximab administration (baseline). Results of rheumatic group compared with the results of healthy control group. After six months from the last rituximab dosage control, TCD performed to the rheumatic group (one year period). Values of bilaterally middle cerebral artery (MCA) peak-systolic, end-diastolic and mean blood flow velocities, Gosling pulsatility index and Pourcelot's resistance index were recorded with TCD by a neurosonologist. The obtained values were compared with each other. The study was approved by the local research ethics committee and all participants gave written informed consent.

of TCD ultrasonography was performed with
Multi-Dop X DWL, TCD machine after the subjects rested in a supine position for $10 \mathrm{~min}$. The right and the left MCA were insonated from the temporal windows at 50-65 $\mathrm{mm}$ depth with $2 \mathrm{MHz}$ pulsed Doppler probes. Bilateral MCA peak-systolic, enddiastolic, and mean blood flow velocities; pulsatility index values, and resistance index values were recorded.

\section{Statistical Analysis}

The data was analyzed using the Statistical Package for Social Sciences (SPSS Inc, Chicago, IL, USA) 15.0 program. Paired t-test, cross-tabs tests were used for statistical analysis. In the analysis, $p$ value $<$ 0.05 was considered significant.

\section{RESULTS}

One patient was male and six patients were female. The mean age was found 52.3 years (Table 1). TCD values were compared with rheumatic patients and healthy group (sex and age matched). Then, TCD values of before and after treatment with rituximab compared in each other. Peak-systolic, end-diastolic and mean blood flow velocities of rheumatic patients were significantly higher than healthy group ( $p<$ 0.001) (Table 2). Peak-systolic, end-diastolic and mean blood flow velocities of rheumatic patients were significantly decreased after rituximab therapy $(p<$ 0.001) (Table 3).

\section{DISCUSSION}

Recently there is a considerable attention

Table 1. Demographics and biochemical and hemodynamic characteristics of patients

\begin{tabular}{lccc}
\hline & $\begin{array}{c}\text { Rheumatic group } \\
(\mathbf{n}=7)\end{array}$ & $\begin{array}{c}\text { Control group } \\
(\mathbf{n}=7)\end{array}$ & $p$ value \\
\hline Gender (male/female) & $1 / 6$ & $1 / 6$ & - \\
Age (years) & 52 & 50 & - \\
BMI (kg/m ${ }^{2}$ ) & 22 & 22 & - \\
Systolic Blood Pressure (mmHg) & 130 & 125 & 0.22 \\
Diastolic Blood Pressure & 80 & 80 & 0.33 \\
(mmHg) & & & $<0.001$ \\
CRP (mg/L) & 6.8 & 1.47 & \\
\hline
\end{tabular}

$\mathrm{BMI}=$ body mass index, $\mathrm{CRP}=\mathrm{C}$-reactive protein 
Table 2. Transcranial Doppler data of rheumatic group compared with healthy control group

\begin{tabular}{lccc}
\hline & $\begin{array}{c}\text { Rheumatic group } \\
(\mathbf{n}=\mathbf{7})\end{array}$ & $\begin{array}{c}\text { Control group } \\
(\mathbf{n}=\mathbf{7})\end{array}$ & $\boldsymbol{p}$ value \\
\hline L-peak systolic BFV & $168.7 \pm 6.8$ & $104.5 \pm 13$ & $<0.001$ \\
L-end diastolic BFV & $62.4 \pm 11.6$ & $38.4 \pm 6.2$ & $<0.001$ \\
L-mean BFV & $106 \pm 13.8$ & $61.8 \pm 8.2$ & $<0.001$ \\
L-PI & $0.87 \pm 0.09$ & $0.9 \pm 0.09$ & 0.78 \\
L-RI & $0.61 \pm 0.03$ & $0.6 \pm 0.05$ & 0.57 \\
R-peak systolic BFV & $157 \pm 7.2$ & $103.5 \pm 17.05$ & $<0.001$ \\
R-end diastolic BFV & $65.7 \pm 13$ & $36.7 \pm 12.36$ & $<0.001$ \\
R-mean BFV & $97.7 \pm 15.47$ & $63.2 \pm 10.5$ & $<0.001$ \\
R-PI & $0.9 \pm 0.08$ & $0.85 \pm 0.09$ & 0.89 \\
R-RI & $0.6 \pm 0.04$ & $0.63 \pm 0.06$ & 0.63 \\
\hline
\end{tabular}

$\mathrm{BFV}=$ blood flow velocity, $\mathrm{L}=$ left, $\mathrm{PI}=$ pulsatility index, $\mathrm{R}=$ right, $\mathrm{RI}=$ resistance index

concerning the possible causal role of systemic inflammation in the development of endothelial dysfunction and accelerated atherosclerosis in rheumatic patients [4, 5]. In these patients, inflammatory markers such as particularly interleukin6 (IL-6), tumor necrosis factor-alpha (TNF- $\alpha$ ) and C-reactive protein (CRP) are found in high levels. Currently, apart from traditional vasculo-protective agents, biologics such as rituximab, infliximab and etanercept may also exert favorable effects on the vasculature but there are conflicting data regarding the effects of these agents on atherosclerosis and arterial stiffness. We aimed to assess the effects of rituximab treatment on inflammation regarding atherosclerosis, a predictor of cerebrovascular diseases, in rheumatic patients.

There may be differences in the effects of TNF blockers on dyslipidemia, as long-term infliximab therapy may be proatherogenic, while some studies suggest that etanercept and adalimumab may exert beneficial effects on lipids. Infliximab stimulates the number and differentiation of endothelial progenitor cells that lead to vascular repair. Preliminary data suggest that rituximab may also improve endothelial function and dyslipidemia [6, 7]. In a study of Provan et al. [8], changes in cardiovascular disease risk markers over the 12-month follow-up in patients receiving rituximab were assessed by mixed models repeated analyses. And they showed significant reductions in these markers [8]. But in another study arterial stiffness did not improve after 6 and 12 months of rituximab therapy. The treatment had a beneficial effect on biologic inflammation and disease activity, but caused a pro-atherogenic lipid profile [9]. Further studies are needed to determine the net effects of biologics on the vasculature.

We found peak-systolic, end-diastolic and mean blood flow velocities were significantly higher in

Table 3. Comparison of TCD data before and after rituximab administration

\begin{tabular}{|c|c|c|c|}
\hline & Before Rtx treatment & $\begin{array}{c}\text { After } 6 \text { months from last Rtx } \\
\text { treatment }\end{array}$ & $p$ value \\
\hline L-peak systolic BFV & $168.7 \pm 6.8$ & $96.57 \pm 4.89$ & $<0.001$ \\
\hline L-end diastolic BFV & $62.4 \pm 11.6$ & $35.85 \pm 4.33$ & $<0.001$ \\
\hline L-mean BFV & $106 \pm 8.83$ & $58.7 \pm 6.89$ & $<0.001$ \\
\hline L-PI & $0.87 \pm 0.09$ & $0.81 \pm 0.06$ & 0.22 \\
\hline L-RI & $0.61 \pm 0.03$ & $0.6 \pm 0.04$ & 0.33 \\
\hline R-peak systolic BFV & $157 \pm 7.2$ & $97.42 \pm 11.2$ & $<0.001$ \\
\hline R-end diastolic BFV & $65.7 \pm 14.6$ & $34.8 \pm 13.8$ & $<0.002$ \\
\hline R-mean BFV & $97.7 \pm 16.45$ & $62.8 \pm 10.9$ & $<0.001$ \\
\hline R-PI & $0.9 \pm 0.08$ & $0.8 \pm 0.08$ & 0.06 \\
\hline R-RI & $0.6 \pm 0.04$ & $0.6 \pm 0.05$ & 0.06 \\
\hline
\end{tabular}


rheumatic group than in the healthy control group. But the pulsatility index and resistance index values were not significantly different in each group. However, correct interpretation of the pulsatility index is complex, because it depends not only on cerebrovascular resistance, but also on several systemic and cerebral variables [10]. The Rotterdam study reported that increased mild-to-moderate cerebral blood flow velocity is due to diffuse atherosclerosis or vasoconstriction [11]. Therefore, mechanisms that underlie this condition (increased velocities but normal PI values) are most likely to be due to mild diffuse subclinical atherosclerosis.

\section{The Limitations of the Study}

Our study had methodological limitations, including inadequate sample size and non-blinding of those involved in the measurements.

\section{CONCLUSION}

Increased cerebral blood flow velocities were observed in patients with rheumatologic diseases. The normalization of increased blood flow velocity with rituximab treatment suggests a reversible condition.

\section{Conflict of interest}

The authors disclosed no conflict of interest during the preparation or publication of this manuscript.

\section{Financing}

The authors disclosed that they did not receive any grant during conduction or writing of this study.

\section{Acknowledgement}

The paper has been presented as a poster at Annual European Congress of Rheumatology, 10-13 June 2015, EULAR 2015 Roma, Italy.

\section{REFERENCES}

[1] Ross R. Atherosclerosis -- an inflammatory disease. N Engl J Med 1999;340:115-26.

[2] Libby P, Ridker PM, Maseri A. Inflammation and atherosclerosis. Circulation 2002;105:1135-43.

[3] Selmi C, Generali E, Massarotti M, Bianchi G, Sciré CA. New treatments for inflammatory rheumatic disease. Immunol Res 2014;60:277-88

[4] Yüksel S, Ayvazyan L, Gasparyan AY. Familial mediterranean fever as an emerging clinical model of atherogenesis associated with lowgrade inflammation. Open Cardiovasc Med J 2010;23:4:51-6.

[5] Lin CW, Huang YP, Chiu YH, Ho YT, Pan SL. Increased risk of ischemic stroke in young patients with ankylosing spondylitis: a population-based longitudinal follow-up study. PloS One 2014;9:e94027.

[6] Kerekes G, Soltesz P, Der H, Veres K, Szabo Z, Vegvari A, et al. Effects of biologics on vascular function and atherosclerosis associated with rheumatoid arthritis. Ann N Y Acad Sci 2009;1173:814-21.

[7] Szekanecz Z, Kerekes G, Soltesz P. Vascular effects of biologic agents in RA and spondyloarthropathies. Nat Rev Rheumatol 2009;5:677-84.

[8] Provan SA, Berg IJ, Hammer HB, Mathiessen A, Kvien TK, Semb AG. The impact of newer biological disease modifying anti-rheumatic drugs on cardiovascular risk factors: a 12-month longitudinal study in rheumatoid arthritis patients treated with rituximab, abatacept and tociliziumab. PloS One 2015;10:e130709.

[9] Mathieu S, Pereira B, Dubost JJ, Lusson JR, Soubrier M. No significant changes in arterial stiffness in RA after 6 months and 1 year of rituximab treatment. Rheumatology (Oxford) 2012;51:1107-11.

[10] de Riva N., Budohoski KP, Smielewski P, Kasprowicz M, Zweifel C, Steiner LA, et al. Transcranial Doppler pulsatility index: what it is and what it isn't. Neurocrit Care 2012;17:58-66.

[11] Bos MJ, Koudstaal PJ, Hofman A, Witteman JC, Breteler MM. Transcranial Doppler hemodynamic parameters and risk of stroke: the Rotterdam study. Stroke 2007;38:2453-8. 